\title{
GESTIÓN AMBIENTAL EN LA ENSEÑANZA DE LA QUÍMICA ANALÍTICA
}

\author{
Elizabeth Norma Espinosa Descalzo*
}

\begin{abstract}
RESUMEN
El objetivo del presente artículo es generar reflexión respecto al compromiso docente de actualizarse en la problemática ambiental e introducir la asignatura Educación Ambiental en el desarrollo de los principales contenidos teóricos y prácticos de los cursos de la Escuela Profesional de Química en la formación de futuros profesionales quienes, además de dominar la ciencia, dentro de su campo y rango de acción, profundicen con el suficiente rigor, el compromiso con el ambiente, además de minimizar las emisiones nocivas que sus actividades generan, para contribuir al desarrollo sostenible. Una forma de incluir los principios de la Educación Ambiental, es mediante la realización de una gestión participativa en las prácticas de laboratorio, en base al principio de reducción en la fuente que, además de ayudar en la preservación ambiental, puede generar beneficios económicos como la disminución del consumo de reactivos del orden del 33,33 al $80 \%$. Asimismo, en el orden formativo, propicia el ejercicio del pensamiento crítico del estudiante y, motiva su participación activa como ciudadano en cuanto a los problemas ambientales, que no sólo son exclusivos al campo de la tecnología, además, tienen el encargo social de formar valores, actitudes y conductas que influyan en las personas y grupos de su entorno.
\end{abstract}

Palabras clave: impacto ambiental, desarrollo sostenible, educación ambiental, gestión ambiental, emisiones nocivas.

\footnotetext{
* Facultad de Química e Ingeniería Química, Universidad Nacional Mayor de San Marcos, Avenida Universitaria s/n, Lima 1, Perú eespinosad@unmsm.edu.pe dirección Jirón Miraflores 252, Lima,15076
} 


\title{
ENVIRONMENTAL MANAGEMENT IN THE TEACHING OF ANALYTICAL CHEMISTRY
}

\begin{abstract}
This article was written with the objective of stimulating reflection on the commitment of teachers to be updated on environmental issues and introduce the Environmental Education subject in the development of the main theoretical and practical contents of the Professional School Chemistry courses on the training of future professionals. These individuals besides of mastering science in their field and range of action, should deepen in the commitment to the environment with sufficient rigor, besides minimizing the harmful emissions their activities would generate in order to contribute to sustainable development. One way to include the principles of Environmental Education is through participatory management in laboratory practices based on the principle of source reduction which, in addition to helping environmental preservation, can generate economic benefits such as reduction in reagent consumption of order 33.33 to $80 \%$. Furthermore, it fosters the exercise of the critical thinking of the student and motivates their active participation as a citizen in terms of environmental problems, which are not only exclusive to the field of technology, but it also has the social responsibility of forming values, good habits and behaviors that influence people around them.
\end{abstract}

Key words: environmental impact, sustainable development, environmental education, environmental management, harmful emissions.

\section{INTRODUCCIÓN}

El uso excesivo e inadecuado de los recursos, naturales y sintéticos, como el agua y los químicos, causan graves alteraciones en el medio ambiente, que reducen las posibilidades de vida en sus diversas formas, acelerando el cambio climático, contaminando suelos, aire, agua y ejerciendo una excesiva presión sobre las reservas naturales a una escala no sostenible. A pesar de conocerse las graves consecuencias de tales prácticas, ellas están muy extendidas entre la población en general, y resulta alarmante observar el despilfarro de recursos por parte de estudiantes, profesionales, personal administrativo y hasta docentes, que mantienen una actitud indiferente y negligente ante tal comportamiento.

Un mayor consumo de recursos hídricos y su mal uso, conduce a volúmenes excesivos de efluentes, elevando los costos de adecuación para reúso o disposición final, tanto para las empresas municipales, que tratan el efluente del alcantarillado general a través de las Plantas de Tratamiento de Aguas Residuales (PTAR), como para las particulares, que están obligadas por la legislación ambiental peruana a tratar los efluentes generados por sus actividades productivas, antes de su vertimiento en los reservorios de agua receptores ${ }^{1}$. Sin embargo, 
diversos sectores empresariales se acogen a prórrogas oficiales reiteradas que les permiten seguir con las mismas prácticas que han conducido a los problemas ambientales, como el Decreto Legislativo N. ${ }^{\circ} 1285$ (2016), que flexibiliza la función fiscalizadora del Estado, de manera que la Autoridad Nacional del Agua (ANA) ya no requiere de la previa opinión técnica favorable de las autoridades de los Ministerios del Ambiente (MINAM) y de Salud (MINSA) para otorgar autorizaciones de vertimiento de efluentes; esto implica que las empresas no sean sancionadas por tener o no, autorización de vertimientos, evaluación de impacto ambiental y autorización de uso de agua residual, y contarán con un plazo de nueve años para la adecuación de sus vertimientos a los Estándares de Calidad Ambiental (ECA) y límites máximos permisibles para vertimientos en el alcantarillado general (LMP); por si fuera poco, prohíbe a la ANA efectuar visitas inopinadas a titulares de las autorizaciones de vertimientos, entre otros aspectos ${ }^{2}$.

En el ámbito científico educativo, la Facultad de Química e Ingeniería Química (FQIQ) de la Universidad Nacional Mayor de San Marcos (UNMSM), presenta determinadas limitaciones en sus instalaciones para el desarrollo de las actividades docentes y de investigación, de ellas, una baja eficiencia en los sistemas de ventilación-extracción en el laboratorio del curso Química Analítica Cuantitativa clásica, para controlar la contaminación generada por el uso de sustancias volátiles durante las prácticas, que comprenden, el análisis de muestras de minerales, aleaciones y otras en mayor escala, desde su disolución con ácidos fumantes, hasta su cuantificación, mediante procedimientos gravimétricos y volumétricos.

Además, la mayoría de los métodos analíticos incluidos en las guías de prácticas del curso fueron desarrollados entre 1940-1960, en laboratorios que contaban entonces con mejores sistemas de control de emisiones, mientras que, en estos momentos, los estudiantes y docentes de la facultad, están expuestos a estas emisiones. En varios de los actuales métodos, se ha observado un uso excesivo de reactivos, lo cual contribuye a incrementar la magnitud de los impactos ambientales mencionados, con consecuencias negativas sobre la salud de docentes y estudiantes, además de emplear un mayor gasto en reactivos. Desde un punto de vista analítico, en el caso de las determinaciones gravimétricas, la solubilidad de los precipitados es incrementada por la mayor concentración de sales presentes, a causa de la influencia de la fuerza iónica o efecto salino, causando resultados falsos negativos, además de incrementarse los fenómenos de contaminación de los precipitados por adsorción, oclusión y otros ${ }^{4,5}$.

Considerando los aspectos legales, pedagógicos y éticos, desarrollar las experiencias de laboratorio en las condiciones mencionadas, no estaría contribuyendo a formar químicos respetuosos del ambiente, comprometidos y capacitados para asumir la responsabilidad de reducir los impactos de sus actividades profesionales y de contribuir al logro de los objetivos del desarrollo sostenible, lo cual debería ser prioritario ante el cambio climático acelerado y el desequilibrio de los diversos ecosistemas, cuestión que pone en riesgo evidente a la especie humana y a las diversas formas de vida que de las que depende ${ }^{6}$.

Ante esta situación, la tesis que sustenta este artículo, ha determinado estudiar las posibles causas de los patrones no sostenibles de consumo y la falta de compromiso de la ciudadanía 
para asumir sus obligaciones ambientales, con el propósito de contribuir a mejorar la situación desde su rol de educadora, interpretando el sentido y compartiendo la frase del estadista francés Jacques Chirac (1932-2019) "Nuestro hogar está en llamas y nosotros, mirando a otro lado..."

Como criterio de la autora, avalado por los resultados del trabajo científico desplegado en ese sentido, una posible razón para esta problemática sería la falta de sensibilización y capacitación de los profesionales de las carreras científicas y de las diversas especialidades de ingeniería para realizar una adecuada gestión ambiental de sus procesos mediante actividades concretas que permitan racionalizar el uso de los recursos desde la fuente, incluyendo la modificación de procesos, reducción y/o sustitución de insumos por otros más inocuos, reúso y reciclaje, entre otros ${ }^{7}$.

\section{FUNDAMENTACIÓN TEÓRICA}

En el caso específico de los estudiantes y egresados de las escuelas profesionales de química e ingeniería química, es prioritaria una capacitación profunda para realizar una gestión ambiental de los procesos, dada la relación con el uso y producción de químicos que provocan la generación de efluentes de diverso grado de toxicidad, volumen y concentración.

Para cumplir con el objetivo mencionado, se han consultado fuentes bibliográficas diversas, como las guías elaboradas por la American Chemical Society (ACS) en sus versiones 1990 y 2002, entre otras, cuya finalidad es promover la gestión adecuada de los desechos en los laboratorios químicos, el desarrollo de alternativas para reducir los volúmenes de desechos antes de la disposición final, frente al problema de saturación de los rellenos de seguridad autorizados, los riesgos de fugas desde los mismos, y los probables accidentes durante el traslado de tóxicos, así como la orientación a los químicos en el conocimiento de la legislación y las especificidades en cuanto a su cumplimiento.

Si bien, estas fuentes ${ }^{8,9}$, no proponen soluciones particulares, mencionan algunas alternativas potenciales que son usualmente realizadas en los laboratorios para el manejo de los desechos peligrosos, en tanto que también, enfatizan la importancia y jerarquía de aplicar el principio de reducción en la fuente, como punto de partida para reducir la generación de este tipo de desechos, promoviendo además el reúso y reciclaje de recursos, siempre que sea factible. En otras publicaciones sobre gestión ambiental en laboratorios de enseñanza, por lo general, presentan recomendaciones generales ${ }^{10}$, más no, un procedimiento sistemático y fundamentado para realizar la reducción de los reactivos en los métodos estudiados, haciéndolo mediante la reducción de un porcentaje de algunos reactivos, o del peso de la muestra, además del reemplazo de reactivos tóxicos por otros menos agresivos con el medio. Para cumplir con el compromiso ético de contribuir a mejorar la capacitación de los estudiantes en aspectos ambientales relacionados con el curso Química Analítica Cuantitativa y de su respectivo laboratorio, se inicia las actividades experimentales con enfoque verde, a partir del año 2017, donde se incluyeron los fundamentos de la educación ambiental en las 
actividades del curso, mediante charlas y actividades propias de una gestión ambiental, en base a los principios de la Química Verde ${ }^{11}$. Para ello se hizo una adecuada revisión teórica con su correspondiente parte experimental, mediante los procedimientos analíticos de la guía de prácticas del curso, considerando la trascendencia de enseñar con el ejemplo.

En las clases de laboratorio se estableció la siguiente secuencia de actividades: introducción sobre los principios de la educación ambiental; explicación de los fundamentos analíticos del método en particular, analizando el tipo de muestra, sus propiedades, solubilidad, las condiciones de seguridad para el manejo de los reactivos, entre otros.

Lo novedoso y a la vez necesario del diseño, es que se incorpora un análisis crítico con enfoque ambiental de cada etapa del método por desarrollar, propiciando la intervención general mediante las opiniones de cada estudiante. Se estima teóricamente el volumen mínimo necesario de ácido para la disolución de la muestra, aplicando la estequiometría y suponiendo que la masa total de la muestra correspondería a la sal anhidra del analito, dado que no se conocen exactamente todos los componentes de la misma.

Dicha suposición permite plantear la ecuación química balanceada de la disolución de la masa de muestra con el ácido específico, para obtener los equivalentes gramo y/o pesos fórmula gramo del analito en dicha muestra, el volumen necesario de ácido según su concentración, controlar el exceso adecuado del ácido, para asegurar una reacción de disolución cuantitativa de la muestra. De la misma forma se realiza el cálculo del reactivo requerido para lograr la precipitación cuantitativa del analito, incluyendo el exceso necesario para el desplazamiento del equilibrio en favor del producto, en función de su solubilidad.

Esta actividad ha sido desarrollada por la autora en el laboratorio del curso Química Analítica Cuantitativa de la Facultad de Química e Ingeniería Química de la UNMSM, desde el 2017 al 2020, bajo un enfoque ambiental y formativo, con la participación de los estudiantes de la Escuela Profesional de Química de los grupos a su cargo, como tema central de la preparación de su tesis de maestría en Educación, con mención en Educación Ambiental y aprobada e inscrita como proyecto de tesis en la Unidad de Posgrado de la Facultad de Educación de la UNMSM el 13 de febrero de 2020, habiéndose revisado los métodos analíticos de las siguientes guías de prácticas del curso de los semestres 2017-1, 2018-1, 2019-1 y 2020-1 :

1. Determinación gravimétrica de sulfato en yeso como sulfato de bario (guías 2018-2019).

2. La determinación de sílice, como parte del análisis gravimétrico de una muestra de caliza (guía de 2020-1)

3. La disolución de la muestra de una aleación ternaria de níquel para la determinación gravimétrica de níquel en aleaciones con dimetilglioxima (guía 2018-1).

4. Determinación gravimétrica de ion ortofosfato en fosfatos con molibdato de amonio (guías 2019-1 y 2020-1) 


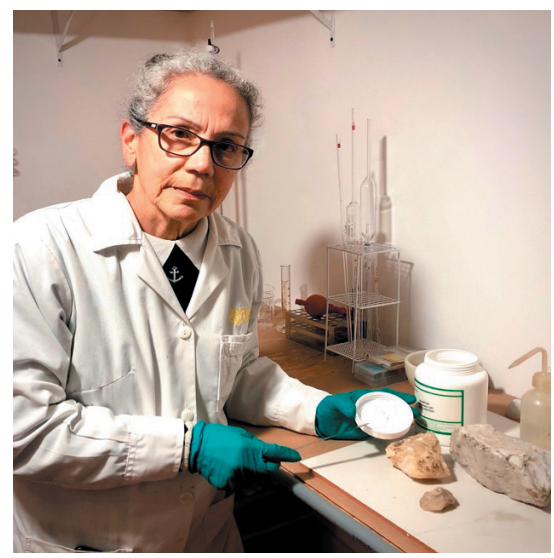

Figura 1. Imagen de la investigadora, presentando tres muestras en bruto de caliza, una de ellas finamente molida.

\section{PARTE EXPERIMENTAL}

Antes de realizar la parte experimental, se efectuaron los cálculos de las modificaciones en las cantidades y procedimientos, según los ejemplos de cálculo que son similares para los métodos enumerados en la sección anterior como 1, 2 y 4.

Cálculo del volumen necesario para disolver en promedio $0,15 \mathrm{~g}$ de la muestra de yeso para la determinación de sulfatos; cuyo componente principal es $\mathrm{CaSO}_{4} \cdot 2 \mathrm{H}_{2} \mathrm{O}(172,14 \mathrm{~g} / \mathrm{pfg})$. En este caso, se asume que el total de la masa de muestra corresponde a sulfato de calcio anhidro, CaSO4, (136,14 g/pfg.) para plantear la reacción estequiométrica de disolución de la muestra, que ya incluye un exceso al considerarla una sal anhidra:

$\mathrm{CaSO}_{4}+2 \mathrm{HCl} \longleftrightarrow \mathrm{CaCl}_{2}+2 \mathrm{H}^{+}+\mathrm{SO}_{4}=$ $136,14 \mathrm{~g} / \mathrm{pfg} . \quad 36,458 \mathrm{pfg} . \mathrm{x} 2=72,916 \mathrm{~g}$

\section{Cálculo estequiométrico:}

$0,15 \mathrm{~g} / 136,14 \mathrm{~g} / \mathrm{pfg}=0,0011 \mathrm{pfg}$ de CaSO 4 reaccionan con 0,0022 pfg. de $\mathrm{HCl}$, luego si 1000 $\mathrm{mL}$ de $\mathrm{HCl}$ contienen 12 moles de $\mathrm{HCl}$

$\mathrm{V} \mathrm{mL}$ de $\mathrm{HCl}$ contienen 0,0022 moles de $\mathrm{HCl} \quad \mathrm{V}=0,1836 \mathrm{~mL}=0,20 \mathrm{~mL}$

Se decide emplear $1 \mathrm{~mL}$ de $\mathrm{HCl} 12 \mathrm{M}$, el cual incluye un exceso con respecto al mínimo de $0,8 \mathrm{~mL}$, aproximadamente, considerando que la masa de muestra podría variar entre $0,12 \mathrm{y}$ 0,20 g. y contener otros constituyentes de menor pfg. que consuman ácido, además de las posibles pérdidas por evaporación durante la disolución.

Para minimizar las emisiones de cloruro de hidrógeno gaseoso al verter el $\mathrm{HCl} 12 \mathrm{M}$ necesario por muestra, un solo estudiante prepara $200 \mathrm{ml}$ de $\mathrm{HCl} 6 \mathrm{M}$, del que, cada uno, 
extrae $2 \mathrm{~mL}$. /muestra, el cual, podría diluirse a $3 \mathrm{M}$, dado que se agregan $25 \mathrm{~mL}$ de agua destilada como parte del medio para este proceso, reduciéndose proporcionalmente el agua por agregar. La temperatura óptima de disolución de la muestra estuvo alrededor de los 50 $60^{\circ} \mathrm{C}$, dada la curva de solubilidad del sulfato de calcio dihidratado $\left(\mathrm{CaSO}_{4} \cdot 2 \mathrm{H}_{2} \mathrm{O}\right)$ a $25^{\circ} \mathrm{C}$ del orden de $0,2358 \mathrm{~g} / 100 \mathrm{~mL}$, alcanzando su mayor solubilidad en agua a $58^{\circ} \mathrm{C}$ y después decae ${ }^{13}$; siendo soluble en ácido clorhídrico diluido. Al disolverse a una temperatura entre $50-60^{\circ} \mathrm{C}$ se previenen las pérdidas de $\mathrm{HCl}$ por volatilización, reduciendo las emisiones al ambiente durante la disolución de la muestra. Igual procedimiento se ha generalizado para el cálculo del ácido necesario por muestra y para cada método, dejando de usar y medir ácidos concentrados y en exceso. En las muestras con contenidos considerables de hierro, aluminio, manganeso u otras sales hidrolizables, se debe emplear un mayor exceso de ácido diluido para prevenir la hidrólisis. Una vez disuelta la muestra, se neutraliza el exceso de ácido con amoniaco $\left(\mathrm{NH}_{3}\right)$ gastando entre 0,7 y $0,8 \mathrm{ml}$ de $\mathrm{NH}_{3} 15 \mathrm{M}$, aproximadamente; luego se agrega el ácido necesario para ajustar a un $\mathrm{pH}$ cercano a 0,3 un volumen de $100 \mathrm{~mL}$, acondicionando el medio para la precipitación con cloruro de bario al $3 \%$ cuyo volumen se estima a partir de los 0,0011pfg de ion sulfato:

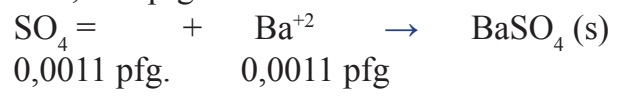

Cálculo del volumen de solución de $\mathrm{BaCl}_{2} \cdot 2 \mathrm{H}_{2} \mathrm{O}$ al $3 \%$; $100 \mathrm{~mL}$ de solución de $\mathrm{BaCl}_{2} \cdot 2 \mathrm{H}_{2} \mathrm{O}$ contienen $3 \mathrm{~g}$ de la sal=3/244,28 g/pfg=0,01228 pfg

Según la guía, se agregan $15 \mathrm{~mL}$ de la solución, equivalente a $0,001842 \mathrm{pfg}$, que representa un exceso de $0,000742 \mathrm{pfg}=0,18 \mathrm{~g}$ de cloruro de bario, en porcentaje $67,47 \%$ mientras que con $10 \mathrm{~mL}$ de igual solución, se obtienen 0,0015 pfg de $\mathrm{Ba}^{+2}$ cuyo exceso se ha considerado suficiente: es mayor que $11,65 \%$ dado que se parte del supuesto de sal anhidra y por la baja solubilidad del precipitado de $\mathrm{BaSO}_{4}$, la cual, en $\mathrm{HCl}$ 0,3 $\mathrm{M}$ es de 2,9mg/100mL, medio ácido necesario para reducir la contaminación del precipitado.

En la revisión de la determinación de sílice del análisis gravimétrico de una caliza, se redujo el volumen de ácido de la forma mencionada, además de modificar el procedimiento: No se lleva a sequedad con el ácido clorhídrico ( 2 veces) para deshidratar el material no soluble en el ácido, en su lugar, se realiza el ataque de la muestra de caliza en condiciones moderadas de temperatura, dado que los carbonatos como el de calcio y magnesio, son solubles en estas condiciones en el ácido usado y, solo se filtra el material no disuelto.

En el método "determinación gravimétrica de níquel en aleaciones con dimetilglioxima", la disolución de la muestra de una aleación ternaria de níquel resulta ineficiente de la forma indicada en la guía de prácticas: "se agregan $15 \mathrm{~mL}$. de $\mathrm{HCl} 1: 1$, se calienta suavemente, hasta disolver, se agregan $3 \mathrm{~mL}$. de ácido nítrico, HNO3 1:1 y se continúa calentando hasta eliminar vapores nitrosos" Lo que sucede es que el $\mathrm{HCl}$ se volatiliza sin disolver la muestra, dada la característica inercia de este tipo de aleaciones. Pero si se agrega la mezcla de los ácidos diluidos clorhídrico y nítrico simultáneamente, se logra un proceso ecoeficiente de disolución. 
En la determinación gravimétrica del ion ortofosfato en un mineral fosfatado con molibdato de amonio, se asume como componente principal al fosfato tricálcico, y por la posible presencia de componentes con menor peso fórmula gramo que dicho fosfato, así como pérdidas por volatilización durante la disolución y reacciones colaterales, se considera un volumen de 2 mL. de $\mathrm{HNO}_{3} 16 \mathrm{M}$, empleando $4 \mathrm{ml}$ del diluido 1:1 (8M) en su reemplazo.

\section{RESULTADOS Y DISCUSIÓN}

Las tablas siguientes, comparan el consumo de reactivos por cada estudiante, que se indica en la guía de prácticas, con las mejoras realizadas, en los métodos analíticos estudiados.

Tabla 1. Consumo de reactivos empleados en la determinación gravimétrica de sulfato en yeso como sulfato de bario, según la guía de prácticas vigente, P1 y de acuerdo a la propuesta de mejora, P2. Masa de muestra promedio $0,15 \mathrm{~g}$ en ambos casos.

\begin{tabular}{|c|c|c|c|c|c|}
\hline $\begin{array}{l}\text { Función del } \\
\text { reactivo }\end{array}$ & $\begin{array}{l}\text { Reactivo } \\
\text { vol. mL }\end{array}$ & P1 & P2 & Ahorro & Ahorro \% \\
\hline $\begin{array}{l}\text { Disolver la } \\
\text { muestra }\end{array}$ & $\begin{array}{l}\mathrm{HCl} \\
12 \mathrm{M}\end{array}$ & $5 \mathrm{~mL}$. & $1 \mathrm{~mL}$ & $4 \mathrm{~mL}$ & 80 \\
\hline $\begin{array}{l}\text { Neutralizar } \\
\text { exceso de } \\
\text { ácido }\end{array}$ & $\begin{array}{l}\mathrm{NH}_{3} \\
15 \mathrm{M}\end{array}$ & $\begin{array}{l}4 \mathrm{~mL} \\
\text { Aprox. }\end{array}$ & $\begin{array}{l}\text { 0,7 mL. } \\
\text { Aprox. }\end{array}$ & $3,3 \mathrm{~mL}$ & 82,5 \\
\hline $\begin{array}{l}\text { Ajuste de } \\
\mathrm{pH}\end{array}$ & $\mathrm{HCl} 12 \mathrm{M}$ & $2 \mathrm{~mL}$ & $2 \mathrm{~mL}$ & $0 \mathrm{~mL}$ & 0 \\
\hline $\begin{array}{l}\text { Agente } \\
\text { precipitante }\end{array}$ & $\begin{array}{l}\mathrm{BaCl}_{2} \text { al } \\
3 \%\end{array}$ & $15 \mathrm{~mL}$ & $10 \mathrm{~mL}$ & $5 \mathrm{~mL}$ & 33,33 \\
\hline
\end{tabular}

Tabla 2. Consumo de reactivos para la determinación de material insoluble en ácido incluido en el análisis de una muestra de caliza. De acuerdo a la guía actual P3, según la propuesta, P4.

Masa promedio de muestra $0,5 \mathrm{~g}$. Reducción de ácido y modificación del procedimiento.

\begin{tabular}{llllll}
\hline $\begin{array}{l}\text { Función } \\
\text { del reactivo }\end{array}$ & $\begin{array}{l}\text { Reactivo } \\
\text { vol. mL }\end{array}$ & $\mathrm{P} 3$ & $\mathrm{P} 4$ & $\begin{array}{l}\text { Ahorro en } \\
\text { reactivos }\end{array}$ & Ahorro \% \\
\hline $\begin{array}{l}\text { Disolver la } \\
\text { muestra }\end{array}$ & $\mathrm{HCl} 6 \mathrm{M}$ & $10 \mathrm{~mL}$ & $4 \mathrm{~mL}$ & $6 \mathrm{~mL}$ & 60 \\
\hline $\begin{array}{l}\text { Deshidratar } \\
\text { ácido } \\
\text { silícico }\end{array}$ & $\mathrm{HCl} 6 \mathrm{M}$ & $11,2 \mathrm{~mL}$ & $0 \mathrm{~mL}$ & $11,2 \mathrm{~mL}$ & 100 \\
\hline $\begin{array}{l}\text { Neutralizar } \\
\text { exceso } \mathrm{HCl}\end{array}$ & $\mathrm{NH}_{3} 15 \mathrm{M}$ & $0,8-1 \mathrm{~mL}$ & $0,8-1 \mathrm{~mL}$ & $0 \mathrm{~mL}$ & 0 \\
\hline
\end{tabular}


Tabla 3. Consumo de reactivos en la disolución de $0,1 \mathrm{~g}$ de una aleación ternaria de níquel (alpaca, $\mathrm{Zn}, \mathrm{Cu}$ y Ni), en el método determinación gravimétrica de níquel en aleaciones con dimetilglioxima, según procedimiento de la guía, P5 y según propuesta P6.

\begin{tabular}{llllll}
\hline $\begin{array}{l}\text { Función } \\
\text { del reactivo }\end{array}$ & $\begin{array}{l}\text { Reactivo } \\
\text { vol. } \mathrm{mL}\end{array}$ & P5 & P6 & $\begin{array}{l}\text { Ahorro en } \\
\text { reactivos }\end{array}$ & Ahorro \% \\
\hline $\begin{array}{l}\text { Disolver la } \\
\text { muestra } 1^{\mathrm{a}}\end{array}$ & $\begin{array}{l}\mathrm{HCl} \\
\text { adición }\end{array}$ & $15 \mathrm{~mL}$ & $\begin{array}{l}\text { Mezcla } \\
\text { de } \mathrm{HCl} \text { y } \\
\mathrm{HNO} \text { en }\end{array}$ & $\begin{array}{l}\text { Evita la } \\
\text { pérdida de }\end{array}$ & $\begin{array}{l}100 \% \text { de } \\
\text { tiempo de la }\end{array}$ \\
\hline $\begin{array}{l}1^{\mathrm{a}} \text { adición } \\
1^{\mathrm{a}} \text { adición }\end{array}$ & \\
\hline $\begin{array}{l}\text { Disolver la la } \\
\text { muestra 2 } \\
\text { adición }\end{array}$ & $\begin{array}{c}\mathrm{HCl} \\
6 \mathrm{M}\end{array}$ & $10 \mathrm{~mL}$ & $0 \mathrm{~mL}$ & $10 \mathrm{~mL}$ & 100 \\
\hline $\begin{array}{l}\text { Disolver la } \\
\text { muestra }\end{array}$ & $\begin{array}{l}\mathrm{HNO} \\
8 \mathrm{M}\end{array}$ & $3 \mathrm{~mL}$ & $3 \mathrm{~mL}$ & $0 \mathrm{~mL}$ & 0 \\
\hline
\end{tabular}

Tabla 4. Consumo de reactivos en la determinación gravimétrica de ion ortofosfato con molibdato de amonio, según procedimiento de la guía, P7 y según propuesta P8. Peso de muestra $0,2 \mathrm{~g}$ en ambos casos.

\begin{tabular}{llllll}
\hline $\begin{array}{l}\text { Función del } \\
\text { reactivo }\end{array}$ & $\begin{array}{l}\text { Reactivo } \\
\text { vol. mL }\end{array}$ & P7 & P8 & Ahorro & Ahorro \% \\
\hline $\begin{array}{l}\text { Disolver la } \\
\text { muestra }\end{array}$ & $\begin{array}{l}\mathrm{HNO}_{3} \\
16 \mathrm{M}\end{array}$ & $5 \mathrm{~mL}$ & $2 \mathrm{~mL}$ & $3 \mathrm{~mL}$ & 60 \\
\hline $\begin{array}{l}\text { Neutralizar } \\
\text { exceso de } \\
\text { ácido }\end{array}$ & $\mathrm{NH}_{3} 15 \mathrm{M}$ & $\begin{array}{c}4 \mathrm{~mL} \\
\text { Aprox. }\end{array}$ & $\begin{array}{c}1 \mathrm{~mL} \\
\text { Aprox. }\end{array}$ & $3 \mathrm{~mL}$ & 75 \\
\hline $\begin{array}{l}\text { Ajuste de } \\
\text { pH }\end{array}$ & $\begin{array}{l}\mathrm{HNO}_{3} \\
16 \mathrm{M}\end{array}$ & $0,5 \mathrm{~mL}$ & $0,5 \mathrm{~mL}$ & $0 \mathrm{~mL}$ & 0 \\
\hline
\end{tabular}

Tabla 5. Resultados analíticos, determinación gravimétrica de sulfato.

\begin{tabular}{lllll}
\hline Semestre & $2017-1$ & $2018-1$ & $2019-1$ & $2019-1$ \\
\hline Procedimiento & Guía & Propuesto & Propuesto & Guía \\
\hline Docente & Espinosa & Espinosa & Espinosa & Rodríguez \\
\hline & 53,31 & 52,04 & 54,33 & 56,35 \\
& 54,38 & 56,20 & 55,50 & 51,93 \\
& 51,72 & 47,24 & 53,79 & 51,18 \\
& 55,13 & 61,42 & 54,38 & 56,98 \\
& 43,96 & 55,23 & 55,39 & 54,77 \\
& 53,62 & 53,64 & 53,78 & 54,15 \\
& 59,64 & 55,35 & 56,80 & 56,44 \\
& 53,11 & 54,26 & 54,38 & 57,88 \\
\hline Promedio & 53,11 & 54,42 & 54,79 & 54,96 \\
\hline $\begin{array}{l}\text { Desviación } \\
\text { estándar }\end{array}$ & $\mathbf{4 , 3 8 0 0}$ & $\mathbf{3 , 9 9 1 8}$ & $\mathbf{1 , 0 3 3 2}$ & $\mathbf{2 , 4 1 6 9}$ \\
\hline
\end{tabular}




\section{DISCUSIÓN DE RESULTADOS}

1.- Respecto a los métodos estudiados y optimizados en el presente, los estimados teóricos de volúmenes de ácidos para disolver las muestras, han estado dentro de lo esperado, habiéndose comprobado experimentalmente en la práctica, dado que se sustentan en la estequiometría y la suposición de asumir la composición de la muestra como la de su sal anhidra. Los resultados de la tabla 5 corresponden a muestras de yeso analizadas por los estudiantes desde el 2017, siguiendo el procedimiento de la guía tradicional, y en 2018 y 2019 aplicando ambos procedimientos, (el de la guía y los propuestos), se puede ver que los mejores resultados (menor desviación) corresponden a 2019-1 optimizados en base a las experiencias anteriores. Los resultados de los métodos 2, 3 y 4 se consideran preliminares, en vista que corresponden a un solo semestre y a un grupo, no se han presentado, por no disponerse de resultados referenciales de los otros grupos, quedando pendiente continuar con estos ensayos.

2.-Se han logrado minimizar las emisiones de cloruro de hidrógeno, gases nitrosos y amoniaco en todas las operaciones de las prácticas, al emplear ácidos diluidos, de manera que, al manipularlos no se liberen vapores al ambiente, incluyendo el proceso de disolución realizado en condiciones de temperatura mínima, basado en la curva de solubilidad del sulfato de calcio (solubilidad máxima en agua $58^{\circ} \mathrm{C}$ ). Disolviendo a temperaturas entre 50 $60^{\circ} \mathrm{C}$ se minimizan las pérdidas de $\mathrm{HCl}$ por volatilización. Igualmente, en la determinación de fosfatos con ácido nítrico y de la aleación ternaria de níquel que no se solubiliza sólo en $\mathrm{HCl}$, ya que requiere de la mezcla de ácidos $\mathrm{HCl}$ y $\mathrm{HNO} 3$ diluidos, desde el inicio del proceso, evitándose agregar más $\mathrm{HCl}$ para reponer las pérdidas, además de reducir el tiempo de disolución en un $100 \%$.

3.-Al reducir los ácidos para disolución de muestras, en porcentajes entre 60 y $80 \%$, se logra ahorrar en la neutralización de los ácidos en exceso, con hidróxido de amonio NH3, alrededor del $80 \%$ de éste y, ajustar el $\mathrm{pH}$ para prevenir las interferencias y minimizar los fenómenos de contaminación de los precipitados.

4.-Además, al emplear menos ácidos y amoniaco, se reduce la concentración salina en el licor madre, disminuyendo las consecuencias desfavorables del "efecto salino" para las técnicas gravimétricas, dado que incrementa la solubilidad y los fenómenos de impurificación de los precipitados.

5.-Asimismo, la disminución de sales, favorece un proceso de filtración y lavado del precipitado y papel de filtro más eficiente, debido a la menor viscosidad del licor madre al tener menos sólidos disueltos, se reduce el tiempo de filtración y lavado al $50 \%$ del tiempo. 6.-De acuerdo con la guía de prácticas, en la disolución del mineral fosfatado con ácido nítrico, puede quedar un residuo insoluble, el cual se filtra sin realizar un tratamiento adicional para disolver los materiales poco solubles; mientras en el caso de la caliza, se recurre a llevar a seco dos veces con $\mathrm{HCl}$ para deshidratar el ácido silícico liberado, empleando más $\mathrm{HCl}$ y tiempo. Dado que, el objetivo del análisis es disolver la caliza para determinar su pureza como $\mathrm{CaCO} 3$, y su rendimiento en la producción de cal $(\mathrm{CaO})$ por $\mathrm{kg}$ de la misma, se 
propone filtrar los insolubles sin mayor tratamiento que la disolución inicial, lo cual ahorra reactivos, tiempo, y contribuye positivamente a reducir el impacto ambiental.

\section{CONCLUSIONES}

El procedimiento propuesto, es una forma sistemática para estimar los requerimientos mínimos de reactivos en un método analítico y, puede ser aplicado para optimizar, desarrollar e implementar diversos métodos analíticos, disminuir costos en reactivos y reducir los impactos ambientales que implica un consumo mayor del necesario.

También, el conocimiento adquirido permite a los estudiantes comprobar la validez de los estimados teóricos, además de demostrarles que es posible "hacer más con menos": ser eco eficientes, cuestión que permite realizar una gestión ambiental adecuada, que, como futuros profesionales químicos, incidirá en disminuir los impactos de las actividades productivas en las que participe, contribuyendo, entre otras cosas, al cuidado de la salud pública.

Por otra parte, es posible reducir sustancialmente la emisión de gases y vapores durante las prácticas, al realizar cambios sencillos, como usar ácidos diluidos en lugar de concentrados, realizar las operaciones en condiciones más suaves y eficientes, sin mayor inversión de capital, traducidas en ahorro que podría ser invertido en mejorar las instalaciones del laboratorio, referidas anteriormente.

Mediante los resultados de los estudios que se han mostrado, la autora demuestra la necesidad de impartir educación ambiental transversalmente, en todos los cursos, tanto teóricos como prácticos, con enfoque en el conocimiento de los problemas reales de cada campo profesional como un aspecto esencial para promover la formación integral de profesionales que sean agentes de cambio, y líderes capaces de asumir su responsabilidad ambiental en el ámbito de las actividades educativas y su proyección hacia las productivas.

\section{AGRADECIMIENTO}

Mi mayor reconocimiento y agradecimiento al Ingeniero Jorge Eduardo Loayza Pérez por su apoyo constante y desinteresado durante la elaboración de las principales partes de la tesis de maestría. Al Ingeniero Fernando Anaya Meléndez, coordinador del Departamento de Química Analítica en 2018, por darme las facilidades para realizar la revisión de los métodos analíticos. A la Química María Angélica Rodríguez Best, por compartir los resultados analíticos de sus estudiantes, quienes aplicaron las instrucciones de la guía y me apoyaron en las encuestas de referencia.

Al Ingeniero Manuel Yáñez y a mi querida hija Angélica por el invalorable e incondicional apoyo de siempre. 


\section{REFERENCIAS BIBLIOGRÁFICAS}

1. MINAM. Ley de Recursos Hídricos, $\mathrm{N}^{\circ}$ 29338. [Internet] 2009, artículos 75,76,79,80-82 [Citado 12 jun 2020]. Disponible en: https://sinia.minam.gob.pe/normas/ley-recursoshidricos- 0

2. Ministerio de Vivienda, Construcción y Saneamiento. Decreto Legislativo No 1285 . [Internet]Lima, 2016. [Citado 22 julio 2020]. Disponible en: http://nike.vivienda.gob. pe/dgaa/Archivos/DL_1285.pdf

3. Guías de prácticas de laboratorio del curso Química Analítica Cuantitativa del Departamento Académico de Química Analítica de la EP Química de la UNMSM, de los semestres 2017-1, 2018-1, 2019-1 y 2020-1. Lima: EP Química de la Universidad Nacional Mayor de San Marcos.

4. Kolthoff I, Sandell E, Meehan E y Bruckenstein S. Quantitative Chemical Analysis. New York: Macmillan Company; 1969.

5. Harris D. Análisis Químico Cuantitativo. Barcelona: Editorial Reverté; 2012.

6. Erazo M, Cárdenas R. Ecología. Impacto de la problemática ambiental actual sobre la salud y el ambiente. Bogotá: ECOE Ediciones; 2015.

7. Alberta Environmental Protection Agency. Waste Minimization Manual for Analytical Laboratories. Edmonton: Alberta Environmental Protection Agency; 1990. AB T5K $2 \mathrm{~J} 6$.

8. American Chemical Society (ACS). Waste Management Manual for Laboratory Personnel. Washington, D.C.: American Chemical Society; 1990.

9. Work Team of the Environment, Health and Safety laboratory and the American Chemical Society (ACS). Less is Better. A Guide for the Minimization of Waste in Laboratories. Washington, D.C.: American Chemical Society; 2002.

10. Loayza J, Silva M, Galarreta H. Gestión Integral de Residuos Químicos en el Laboratorio. Revista Peruana de Ingeniería Química. 2005; 8 (2): 53-60

11. Robustillo A. Estudio de la solubilidad de carbonatos y sulfatos con implicancia en la formación de "Scales". [Tesis de pregrado]. Madrid: Universidad Rey Juan Carlos; 2009.

12. de la Guardia M, Armenta S. Green Analytical Methods. Anal Bioanal Chem. 2012; 404: 625-626. 